\title{
QUALIDADE DE VIDA DOS IDOSOS EM HEMODIÁLISE: RELAÇÃO COM A ESPERANÇA, RELIGIOSIDADE E ESPIRITUALIDADE
}

Fernanda Rosa de Oliveira Pires. Departamento de Enfermagem Universidade Federal de Sant Catarina; nandadode@hotmail.com;

Caroline Bittelbrunn. Departamento de Enfermagem Universidade Federal de Sant Catarina; carolbittelbrun@hotmail.com;

Maria Elena Echevarría-Guanilo. Departamento de Enfermagem da Universidade Federal de Santa Catarina (UFSC); elena_meeg@hotmail.com;

Giordanna Nayara Chagas e Silva; Departamento de Enfermagem da Universidade Federal do Paraná (UFPR); giordanna@ufpr.br;

Danieley Cristini de Lucca. Departamento de Enfermagem da Universidade Federal de Santa Catarina (UFSC); danylucca.enf@gmail.com;

Fernanda Cegan Gribner. Departamento de Enfermagem da Universidade Federal do Paraná (UFPR); fernandacegan@ufpr.br;

Karina Silveira de Almeida Hammerschmidt; Departamento de Enfermagem da Universidade Federal do Paraná (UFPR); ksalmeidah@ufpr.br;

\section{RESUMO}

Introdução: A Doença Renal Crônica (DRC) envolve diversas mudanças na rotina do idoso, que podem refletir na esperança de vida dos pacientes, neste contexto a esperança, espiritualidade e religiosidade podem estar associadas como ferramentas de apoio. Objetivo: Apontar a interferência do tratamento hemodialítico na qualidade de vida de idosos renais crônicos, relacionando os achados com a esperança, espiritualidade e religiosidade dos mesmos, de modo a contribuir para o planejamento de intervenções cabíveis à prática e de acordo com as reais necessidades dos indivíduos. Método: Trata-se de estudo de abordagem quantitativa, do tipo descritivo e correlacional, de temporalidade transversal, realizado em Unidade de Tratamento Dialítico de um Hospital Universitário de Santa Catarina. Foram entrevistados 22 idosos, idade entre 60 e 69 anos e em tratamento há 18 meses (média). Os dados foram obtidos a partir de cinco instrumentos: questionário de caracterização, Kidney Disease and Quality-of-Life (KDQOL), Escala de Esperança de Herth (EEH), Índice de Religiosidade da Duke (DUREL) e Escala de Espiritualidade de Pinto e Pais-Ribeiro (EEPP-R). Resultados: A qualidade de vida estava afetada nos domínios de situação de trabalho, função emocional e nos relacionados à função física; e elevada nos domínios de função cognitiva, suporte social e satisfação com a equipe profissional. A religiosidade estava bem presente e os níveis de esperança de vida e espiritualidade foram altos. Conclusão: $\mathrm{O}$ estudo apontou para a interferência positiva da religiosidade e espiritualidade na qualidade e esperança de vida dos idosos entrevistados.

Palavras-chave: Idoso; Diálise Renal; Qualidade de Vida; Esperança; Espiritualidade. 